\title{
MRI Brain Image Segmentation based on Wavelet and FCM Algorithm
}

\author{
Iraky khalifa \\ Faculty of Computers \& \\ Information, Helwan University, \\ Cairo, Egypt.
}

\author{
Aliaa Youssif \\ Faculty of Computers \& \\ Information, Helwan University, \\ Cairo, Egypt.
}

\author{
Howida Youssry \\ Faculty of Information \\ Technology, Misr university for \\ science and technology, \\ 6th of Oct. City, Egypt
}

\begin{abstract}
Image segmentation plays a preliminary and indispensable step in medical image processing. Magnetic resonance (MR) segmentation used for brain tissues extraction white matter (WM), gray matter (GM) and cerebrospinal fluids (CSF). These tissues help in many medical image segmentation applications such as radiotherapy planning, clinical diagnosis, treatment planning and Alzheimer disease. This paper presents a novel manipulation or utilization of Fuzzy CMeans (FCM) Clustering by using wavelet Decomposition for feature extraction and feature vector treat as input to FCM. This algorithm is called Wavelet Fuzzy C- means (WFCM), the algorithm results are compared with standard FCM and Kernelized Fuzzy C- Means (KFCM). The performance of the proposed segmentation algorithm provides satisfactory results compared with the other two algorithms.
\end{abstract}

\section{General Terms}

Pattern Recognition, image segmentation.

\section{Keywords}

Image segmentation, Fuzzy C-means, Kernel method, kernelinduced distance, magnetic resonance imaging, and Wavelet.

\section{INTRODUCTION}

Image analysis is one of the most significant key steps in medical image segmentation and computer vision. Medical image segmentation results affect significantly on medical image analysis. Medical image segmentation is an essential step in many medical image applications such as radiotherapy planning, clinical diagnosis and treatment planning[1, 2], quantification of tissue volumes, localization of pathology, study of anatomical structure, partial volume correction of functional imaging data, and computer integrated surgery [3]. It's a challenging and an unsolved problem. Image segmentation dividing an image into regions with homogenous properties such as gray level, color, texture, brightness, and contrast, or combines all this information[1, 2, 4].

The rest of the paper is organized as follows. Section 2 Literal review of medical image segmentation, section 3 presents a brief review of the research in Fuzzy C-means (FCM), in Sections 4,a brief review of the research in Kernelized Fuzzy $\mathrm{C}$-means (KFCM) is presented, the presented model of medical image segmentation is described in detail in section 5 . The experimental results and discussions are provided in Section 6. Finally, the conclusions are summed up in Section 7 .

\section{LITERAL REVIEW}

An automatic MR images segmentation is a difficult mission. Because of MR images have difficult natural, also have no linear features. MR image segmentation performance is affected by many issues as Partial Volume Effects (PVE) which means a pixel contains more than one tissue, this leads to misclassification as a result of blurred boundary between tissues, Intensity Non-Uniformity (INU) means that the artifact intensities of the same tissue are not constant over the image spatial domain, and geometric deformations [3, 4]. There are different classifications of medical image segmentation techniques. There are no standard classifications of techniques. The most commonly used segmentation techniques can be classified into two broad categories, regionbased segmentation techniques that look for regions satisfying a given homogeneity criterion, and Edge-based segmentation techniques that look for edges between regions with different characteristics [3]. Region-based techniques depend on partitioning an image into regions according to common image properties, these properties can be intensity values, a texture pattern, a gradient, a colour, brightness, geometric properties and homogeneity properties, based on similarity criterion[5]. Region-based techniques divide into Split and Merge, and Region Growing [6, 7]. Split and Merge based on quad quadrant tree representation, the Split process will be repeated until each region contains only homogeneous pixels $[5,7]$, this lacks of sensitivity to image semantics[8]. Region Growing depends on bottom up technique, it starts with $n$ pixels as seeds, each seed is treated as a region, and then region grows by adding its neighbour pixels with similar properties. Its drawbacks are: difficulty to detect edges, its seed pixels generally require manual input for each region to be segmented, and being affected by partial volume leads to holes or disconnection $[9,10]$. Edge-based Segmentation depends on applying gradient operator edges in the image, so its algorithms have two main steps: edge detection and edge linking. [7, 11-13]. Level Set Method is a counter evolution method which is based on the shape of the contour and driven force field [14-17], it is able to handle the topological change of the regions; but it cannot maintain information of shape. Deformable models idea depends on a boundary of an object such as a parameter for a curve or a surface. A good detection of an object contour is achieved on using a suitable initialization. Deformable model techniques are robust to noise, but they are requiring initialization of a contour that is close to object boundary, which helps to detect true boundary, also it is hard to handle a deformable model as well [18-20].

Clustering is one of the most usable or utilizable techniques in MRI segmentation, where it classifies pixels into classes, without knowing previous information or training. It classifies pixels with highest probability into the same class. It may find unclassified pixels which do not belong to any class 
probability. Clustering techniques training is done by using pixel features with properties of each class [21-23]. This paper will present a brief review of Fuzzy C-means (FCM) and Kernelized Fuzzy C- means (KFCM), as an example of clustering algorithm.

Characteristics of Wavelet Transforms (WT) families share properties of their basis functions, primarily the finite support in both the frequency and original domains, and the scalability. Wavelet families include Haar, Daubechies, Symlets, Coiflets, Biorthogonal , and Reverse biorthogonal ,whereas in this paper Daubechies family is rather used. This family is one of the most used wavelet families in image and signal processing applications such as compression, denoising, classification, and segmentation[8].

\section{VERSION OF FCM}

Fuzzy C- means technique is one of the unsupervised clustering techniques used in image segmentation, its idea depends on clustering data into two or more classes only by known number of classes that image will cluster to it. FCM was first demonstrated by Dunn in 1973 [24] then improved by Bezdek [25] as shown in the following equations :

$$
J_{m}(\mu, v)=\sum_{i=1}^{c} \sum_{j=1}^{n} \mu_{i j}^{m} d^{2}\left(x_{j}, v_{i}\right) \quad \text { subject to } \sum_{i=1}^{c} \mu_{i j}=1
$$

where $X=(x 1, x 2, \ldots x j, \ldots x n)$ is a data matrix with the size of $p \times n, p$ representing the dimension of each $x j$ "feature" vector, and $\mathrm{n}$ represents the number of feature vectors, and vi is the fuzzy cluster centroid of the ith cluster. Using the Euclidean norm, the distance metric d measures the similarity of a feature vector $x j$ and a cluster centroid vi in the feature space. The objective function is minimized when data points close to the centroid of their clusters are assigned high membership values, and low membership values are assigned to data points far from the centroid. Letting the first derivatives of $\mathrm{Jm}$ with respect to $\mu$ and $\mathrm{v}$ equal to zero yields the two necessary conditions for minimizing Jm as follows:

$$
\begin{gathered}
d^{2}\left(x_{j}, v_{i}\right)=\left\|x_{j}-v_{i}\right\|^{2} \\
\mu_{i j}=\left(\sum_{k=1}^{c}\left(\frac{d\left(x_{j}, v_{h}\right)}{d\left(x_{j}, v_{k}\right)}\right)^{2 /(m-1)}\right)^{-1} \\
v_{i}=\frac{\sum_{j=1}^{n} \mu_{i j}^{m} x_{j}}{\sum_{j=1}^{n} \mu_{i j}^{m}}
\end{gathered}
$$

But it has two main drawbacks: 1) it depends on pixel (voxel) intensity of an image but intensity is not trusted in noisy image, 2) the objective function deals with pixels independently and doesn't care about their neighbourhood. Although there are many adopted researches to make FCM algorithm more robust [26]. Most (medications) modifications in FCM are done in the objective function shown in equation (1) as in Robust Fuzzy C-means (RFCM) by Pham 2001 [27], this algorithm changes the objective function of standard FCM by including penalty term . and control by parameter q. Bias Corrected Fuzzy C- Means (BCFCM) by Ahmed et al 2002[28] modified the objective function of standard FCM by using intensity of pixels neighbourhood regularization to solve inhomogeneous intensity. This algorithm open new means to change in FCM algorithm but yet this algorithm consumes a lot of time in segmenting images. Adaptive Spatial Fuzzy C- means (ASFCM) algorithm by Liew and Yan 2003 [29] uses local spatial constraint. Its idea depends on the dissimilarity of local influence of neighbouring pixels such that center pixels on the window is eliminated or smoothed by using membership and cluster centroid computation. Chuang et al 2006 proposed Spatial Fuzzy Cmeans (SFCM) that make modification of standard FCM algorithm by using spatial information in the membership function. The membership function of the neighbours centered on a pixel in the spatial domain is enumerated to obtain the cluster statistics. This statistic is used as a weighing function and using it in the membership function. But This algorithm lacks noise and outliers, and its processing time depends on image size. (and) Its variable ${ }^{\alpha}$ used in the objective function is selected by experience [6]. Cai et al 2007 proposed Fast Generalized Fuzzy C- Means (FGFCM) to overcome drawbacks in Chuang et al algorithm by using local spatial and gray information in a new parameter $\mathrm{Sij}$ similarity measure for both noise which is an elimination and detailpreserving for an image, empirically adjusting parameter $\alpha$. The segmenting time of an image depends on the number of the gray-levels [30]. Wang et al 2008 proposed that the Modified Fuzzy C- Means (MFCM) algorithm. It changes original FCM by using both local and non-local information, and using a dissimilarity index instead of the usual distance metric. However this algorithm has some limitation as it consumes a lot of computation time, and is limited in large $3 \mathrm{D}$ volume data [31].

Sikka et al 2009 presented an algorithm which consists of many steps. First they used entropy driven homomorphic filtering technique which is (has been) employed in this work to remove the bias field. The initial cluster centers used by the proposed algorithm called histogram-based local peak merger using an adaptive window. In Yong's and Chongxun's presented Modified Fuzzy C-mean, an neighbourhood pixel considerations are applied after that the proposed technique. It removes small pixel level noise, which results from misclassified pixels after using the MFCM [32]. Xiao et al 2010 proposed an algorithm by using Gaussian method for smoothing, and homogenizing clustering result and reducing noise. The algorithm uses a weighted clustering scheme that was applied to a Gaussian smoothed image using bootstrapping approach of feature weighting. This algorithm was called Weighted Gaussian FCM (WGFCM) [1]. Xuan et al 2011 proposed Modified Fast Fuzzy C- Means (MFFCM), a method for determining the initial values of the centroids. Then, an adaptive method to incorporate the local spatial is continuity used to overcome the noise effectively and prevent the edge from blurring. The intensity inhomogeneity is estimated by a linear combination of a set of basic functions. A regularization term is added to reduce the iteration steps and accelerate the algorithm. The weights of the regularization terms are all automatically computed to avoid the manually tuned parameter [1].

\section{VERSION OF KFCM}

There are many versions and modifications of Fuzzy Cmeans; one of the most used methods is the Kernel method. The standard FCM algorithm used Euclidean distance measurement, this measure had good results in noise-free data but usually fail in noisy data. In order to overcome this problem some researchers used Kernel methods [1,33]. The Kernel method idea depends on converting low dimensional input space into a higher dimensional feature space through some nonlinear mapping, which helps to overcome a complex linear problems in the low dimensional space [33]. The Kernel 
function's replaced the inner product in the original space, that mapped the space into higher dimensional feature space [34]. So, this leads to modifying the objective function on standard FCM. KFCM methods consist of inducing a class of robust non-Euclidean distance measures for the original data space to derive new objective functions and thus clustering the nonEuclidean structures in data, enhancing robustness of the original clustering algorithms to noise and outliers, and still retaining computational simplicity [35]. The modification on Kernel Fuzzy C- means method is made on the objective function of standard FCM by replacing Euclidean distance with Kernel induced distance is called the Kernelized Fuzzy C-means (KFCM) algorithm [34-39]. The three main types of kernel functions are shown in equations (5, 6 and 7):

Gaussian Radial Basis Function (GRBF) Kernel

$$
K(x, c)=\exp \left(\frac{-\|x-c\|^{2}}{\sigma^{2}}\right)
$$

Polynomial Kernel

$$
K(x, y)=(1+(x, y))^{d}
$$

Sigmoid Kernel

$$
K(x, y)=\tan h(\alpha(x, y)+\beta)
$$

Where $\sigma, d, \alpha, \beta$ are adjustable parameters for Kernel functions. Kernalized Fuzzy C- means by using GRBF Kernel functions are shown in the following equations.

The objective KFCM function is shown in equation (8)

$$
j_{m}=2 \sum_{i=1}^{c} \sum_{j=1}^{N} u_{i j}^{m}\left(1-K\left(x_{j}, c_{i}\right)\right)
$$

Fuzzy membership function is obtained by equation (9)

$$
u_{i j}=\frac{\left(1-K\left(x_{j}, c_{i}\right)\right)^{-1 /(m-1)}}{\sum_{k=1}^{c}\left(1-K\left(x_{j}, c_{k}\right)\right)^{-1 /(m-1)}}
$$

The cluster center can be obtained from equation (10)

$$
c_{i}=\frac{\sum_{j=1}^{N} u_{i j}^{m} K\left(x_{j}, c_{i}\right) x_{j}}{\sum_{j=1}^{N} u_{i j}^{m} K\left(x_{j}, c_{i}\right)}
$$

There are many modifications on KFCM methods as Zhang and Chen 2004 [34, 35] proposed new versions of Ahmed et al algorithm by adding some modifications and named it KFCM_S1, KFCM_S2 respectively that used the extra meanfiltered image and median-filtered image, respectively, which can reduce computation time and also using kernel measurement distance instead of Euclidean distance. But still this algorithm has main (major) drawbacks as the final segmentation results are heavily affected by initial parameter values. Yang and Tsai 2010 [40] proposed Gaussian Kernelbased Fuzzy C-means algorithm (GKFCM) with a spatial bias correction. It is considered as a generalized type of FCM,
BCFCM, KFCM_S1 and KFCM_S2 algorithms, GKFCM can automatically learn the parameters by the prototype-driven learning scheme. Kannan et al 2010 [36] proposed Effective Kernelized Fuzzy C- Means (EKFCM) with weighted bias field Information. Its idea depends on Kernel function and weighted bias field estimation by using center initialization algorithm.

\section{PROPOSED METHOD}

In this paper, we propose an efficient method to segment the MRI brain images. Two major stages are involved in the proposed methodology: feature extraction and clustering. Feature extraction process is performed by using multilevel 2D wavelet decomposition features. The wavelet decomposition outputs are low pass (approximation component) and high pass (detailed components) that horizontal, vertical, diagonal. To obtain the wavelet features, here Daubechies-4(DAUB4) wavelet is applied to the image and performed a two level wavelet transform. Feature extraction from wavelet decomposition is given to Fuzzy CMeans, FCM applied on the feature vector obtained from previous step for clustering. The output image will be segmented into three classes (WM, GM, and CSF) which they are the brain tissue.

\section{Input MR Image}
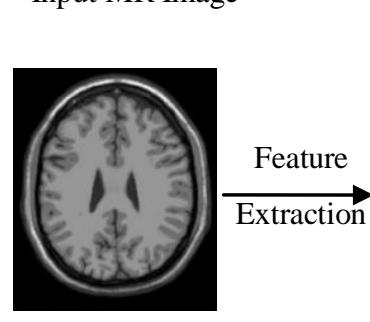

Wavelet Decomposition

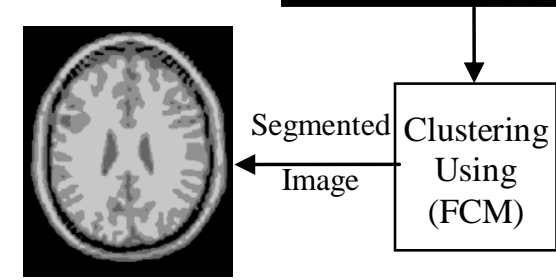

Fig. 1: Diagram of proposed method

\section{DATASET DESCRIPTION}

The proposed Wavelet Fuzzy C- Means (WFCM) algorithm is implemented in Matlab (2010b), and tested on two MRI databases: (1) simulated MRI brain images obtained from BrainWeb and (2) real brain images collected by the Internet Brain Segmentation Repository (IBSR).

\subsection{BrainWeb Database}

The BrainWeb images are simulated MR images generated by the BrainWeb simulator with different level of noise $0 \%, 1 \%$, $3 \%, 5 \%, 7 \%, 9 \%$, and with different level of INU $0 \%, 20 \%$, $40 \%$. These images are obtained from BrainWeb Database at the McConnell Brain Imaging Centre of the Montreal Neurological Institute, McGill University[41] . The used data are T1-weighted, with slice thickness $1 \mathrm{~mm}$, and volume size 
$217 \times 181 \times 181$ images, an example of images is shown in Fig.2 (a and b).

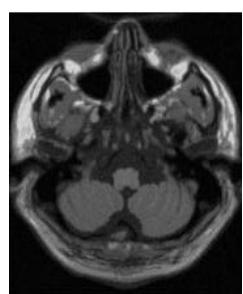

(a)

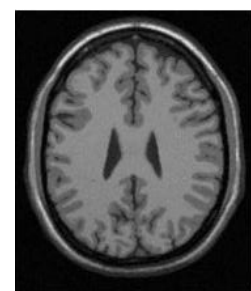

(b)
Fig. 2: (a) T1 simulated brainweb image slice no. \# 20 (b) T1 simulated brainweb image slice no. \# 100

\subsection{IBSR database}

IBSR database is real MRI images collected by Internet Brain Segmentation Repository(IBSR) operated by CMA at MGH, this paper used 20 normal T1-weighted data sets with $1.5 \mathrm{~T}$, $256 \times 256$ section dimension, $60-65$ coronal slice, with slice thickness $3 \mathrm{~mm}$ These datasets contain varying levels of difficulty, with the worst scans consisting of low-contrast and large spatial inhomogeneity [42] . An example of IBSR images as shown in Fig. 3(a, b).

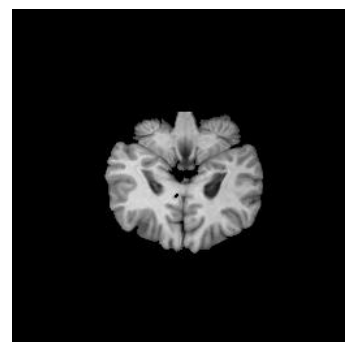

(a)

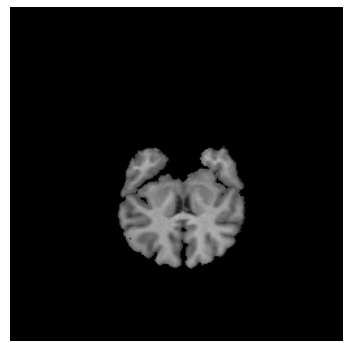

(b)
Fig.3: T1-weighted real MRI image from IBSR:

(a) slice no.\# 23; (b) slice no. \# 33

\subsection{Measuring Segmentation Performance}

The quantitative evaluation of the segmentation performance in this paper is measured by the similarity index between ground truth and segmentation result as shown in equations (11 and12).

Dice Index (Dice) : $D(K)=\frac{2 \cdot V_{p \cap g}(k)}{V_{p}(k)+V_{g}(k)}$,

Where ${ }^{2 . V_{p \cap g}(k)}$ is the number of classified pixels in class $\mathrm{k}$ with two images (a segmented image and a ground truth).

$$
J(K)=\frac{V_{p \cap g}(k)}{V_{p \cup g}(k)}
$$

\subsection{Parameters Tuning}

There are many conditions used in algorithms testing as shown in table 1.
Table1: experimental Parameters

\begin{tabular}{|c|c|c|c|}
\hline $\mathrm{m}^{\text {Algorith }}$ & $\begin{array}{l}\text { Expon } \\
\text { ent }\end{array}$ & $\begin{array}{l}\text { Maximum } \\
\text { number for } \\
\text { clustering } \\
\text { iteration }\end{array}$ & Condition \\
\hline FCM & 2 & 10000 & $1 \mathrm{e}-5$. \\
\hline KFCM & 2 & 10000 & $\begin{array}{l}\text { center_old } \leq \\
\text { center_new }\end{array}$ \\
\hline WFCM & 2 & 10000 & $1 \mathrm{e}-\overline{5}$ \\
\hline
\end{tabular}

\section{EXPERIMENTAL RESULTS}

In BrainWeb database images from $217 \times 181 \times 1$ to $217 \times 181$ $\times 9$ in ground truth images, the pixel values equals zero which it mean to ground truth images to compare results, so there is no segmentation test here, and it is same in slice number from 155 to 181 , so the experimental done from slice number 10 to slice number 154, and no changes were done to simulated image take image as it with no modification. applying the algorithms to simulated BrainWeb images as shown in Fig. $3(\mathrm{a}-\mathrm{c})$ shows results in image with INU $=0 \%$ and different noise level, the results of proposed algorithm indicate a satisfying result even with increasing of noise level comparing to other algorithms. WFCM algorithm indicate stable state and better result in different tissues with different noise level, KFCM is more stable than FCM in different noise level in both GM and WM, but in CSF FCM gives slightly better results. In Fig. 4(a-c) shown a compare performance of three algorithms with $\mathrm{NU}=20 \%$ and different level of noise, which indicate that the proposed algorithm still return satisfy results compare with other two algorithm, FCM is not stable with different level of noise not like KFCM and WFCM. Fig. 5 (ac) shows the results of images with INU $=40 \%$ and a different noise level, the results indicate that the proposed algorithm start with satisfying result in low noise level up to high noise level. An example output image of proposed algorithm results is shown in Fig 6. The brain image in Fig. 6a is a slice of the simulated 3D volume with noise level 9, INU $40 \%$ and slice number 78, the segmentation results of the proposed algorithm WM, GM, CSF are shown in Fig 6b-e, respectively.

The performance of proposed algorithm comparing with other tow algorithm in IBSR database has been shown in Fig. 8(a, b). In WM tissue, WFCM algorithm result was quite good in some image and not good in other images comparing to other two algorithms. In GM tissue was satisfy results in most of images and near to two algorithm results in some images. Sample of segmented image using WFCM is shown in Fig 9. At all our algorithm returns satisfy results comparing to FCM and KFCM even with not satisfy results on WM tissue at IBSR database. 
Volume 47-No.16, June 2012
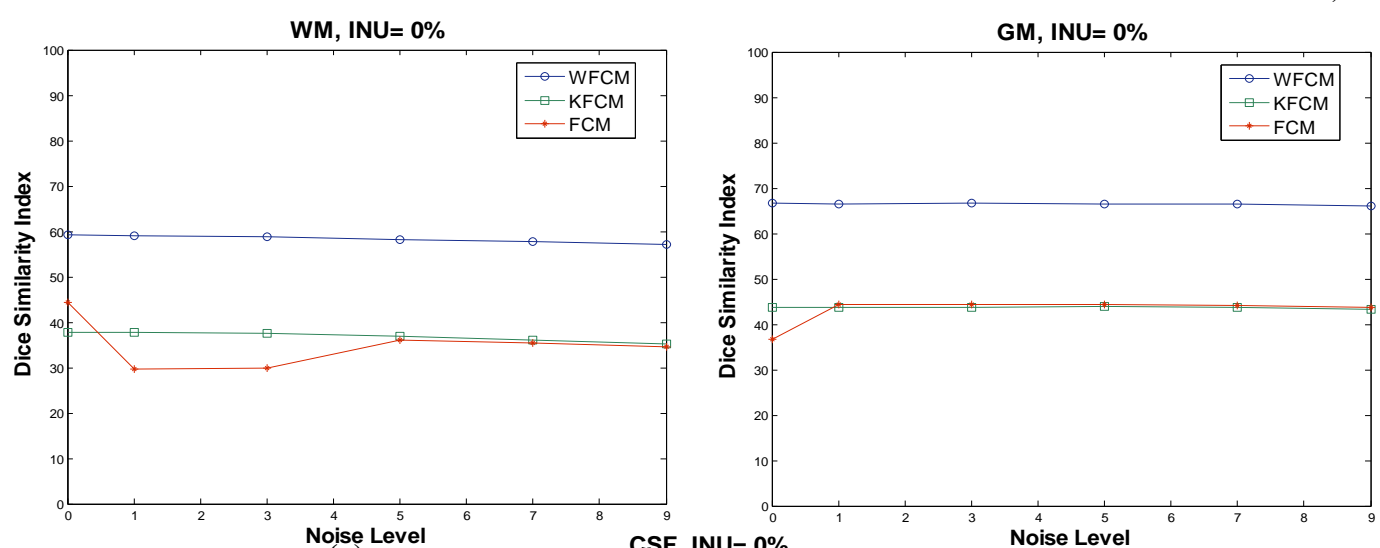

(a)

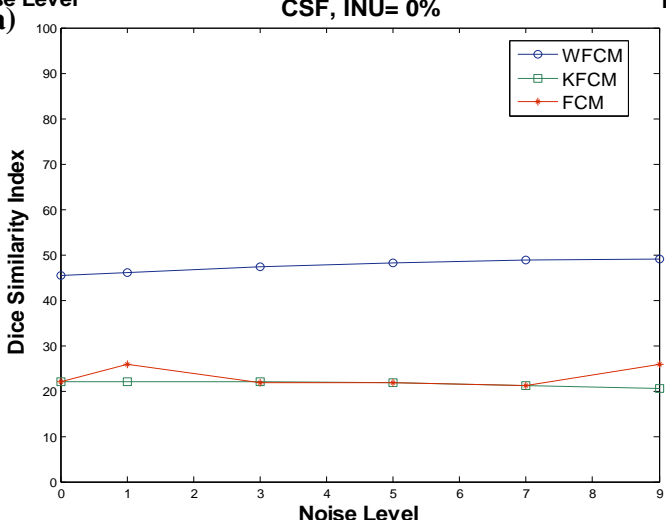

(b)

(c)

Figure 3: Validation results for different noise levels with $0 \%$ INU: (a) white matter; (b) gray matter; (c) cerebrospinal fluid

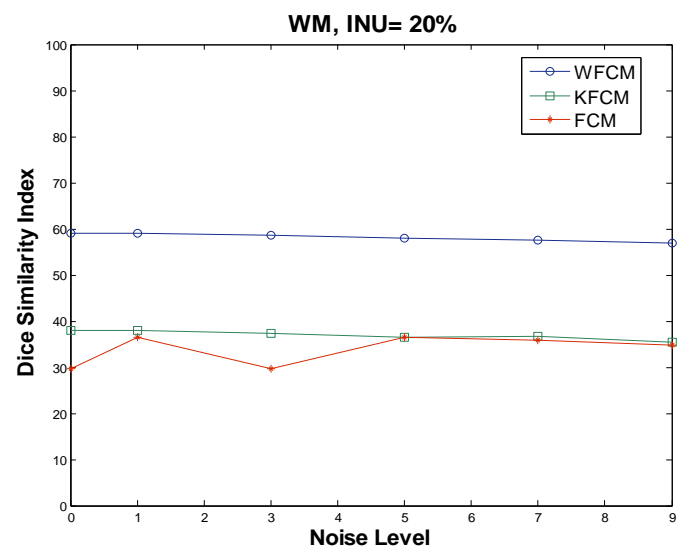

(a)

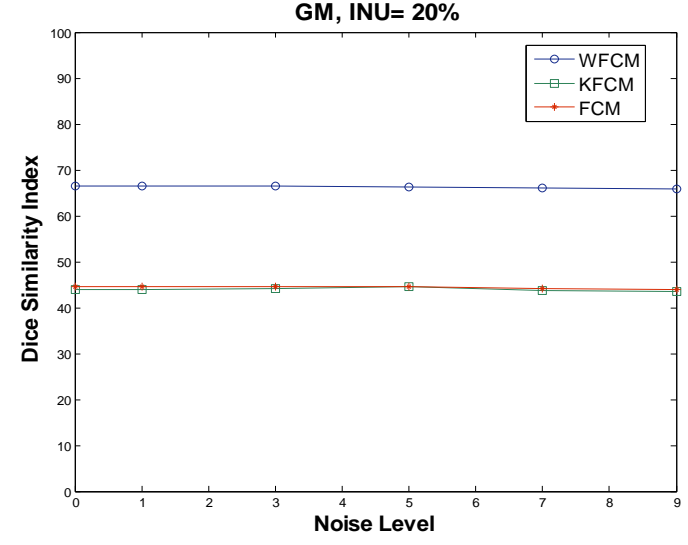

(b)

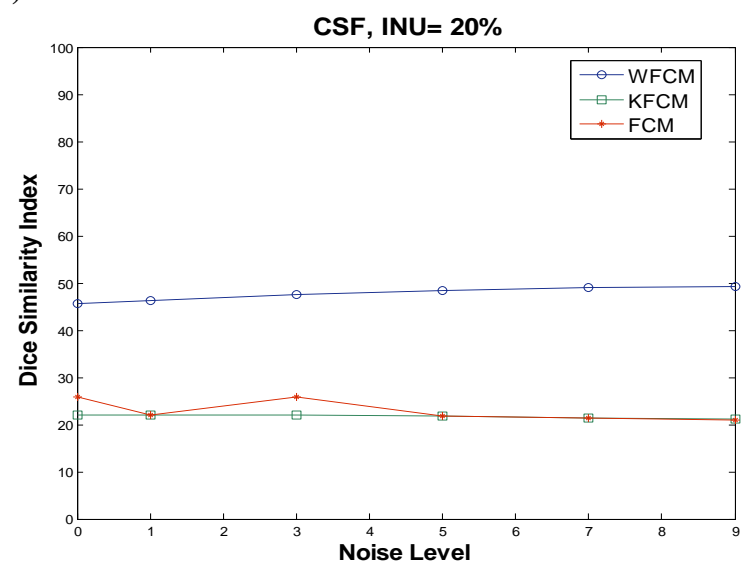

(c)

Fig. 4: Validation results for different noise levels with $20 \%$ INU: (a) white matter; (b) gray matter; (c) cerebrospinal fluid 
Volume 47-No.16, June 2012
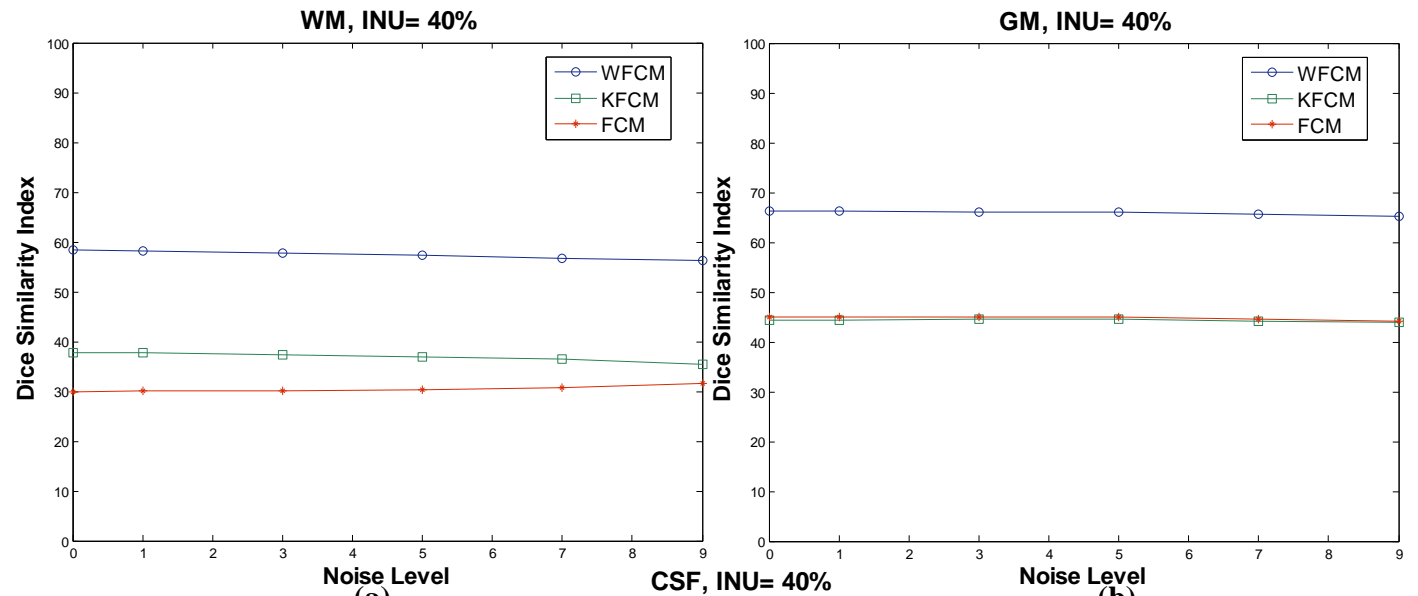

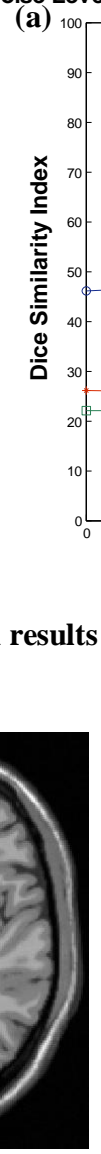

(a)

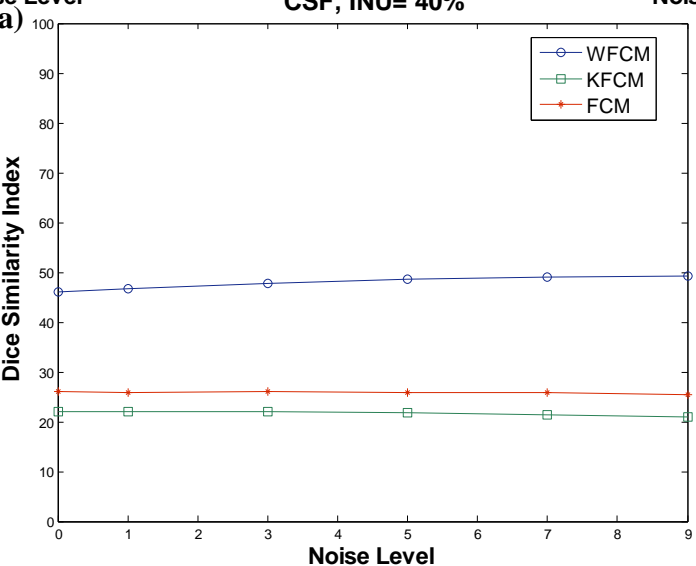

(b)

(c)

Figure 5: Validation results for different noise levels with 40\% INU: (a) white matter; (b) gray matter; (c) cerebrospinal fluid
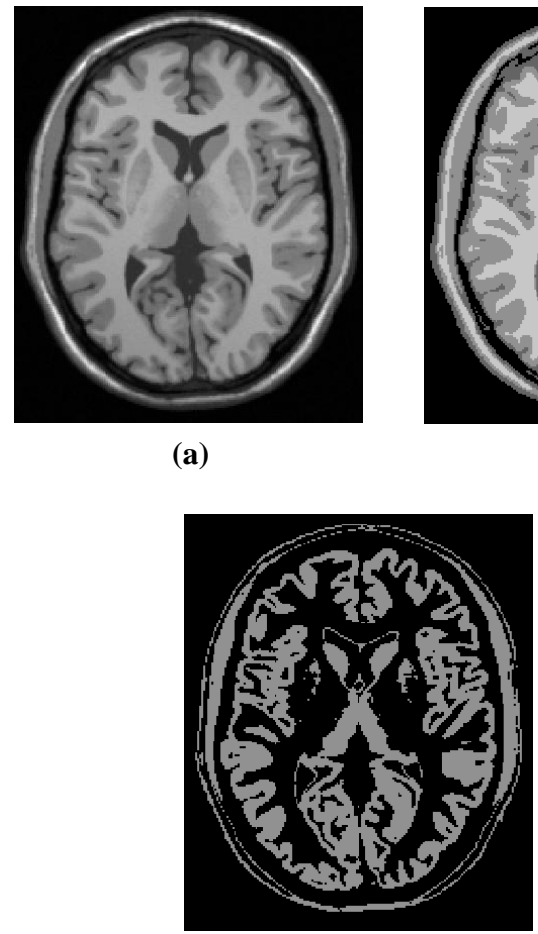

(d)

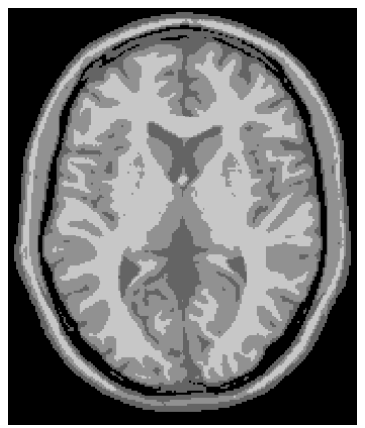

(b)

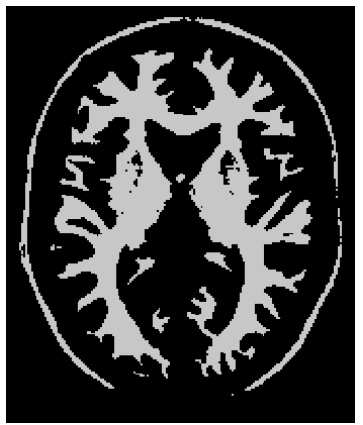

(c)

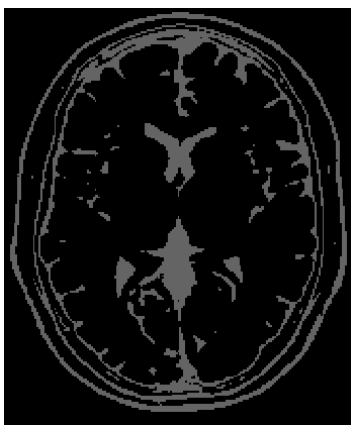

(e)

Figure 6: Segmentation results of the simulated images with proposed algorithm: (a) original image (b) segmented images (c) White Matter (d) Gray Matter (e) CSF 


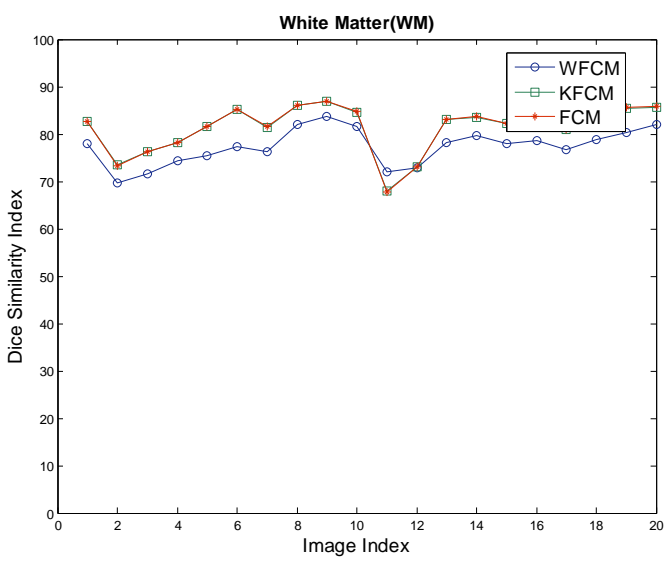

(a)

Figure 8: Validation results for IBSR database

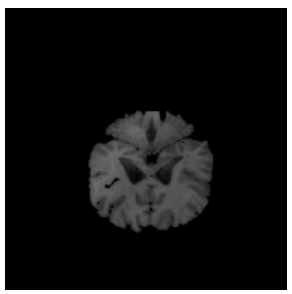

(a)

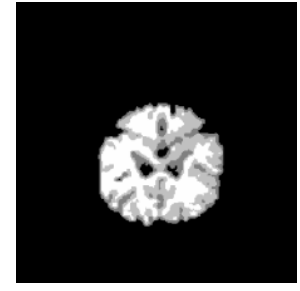

(b)

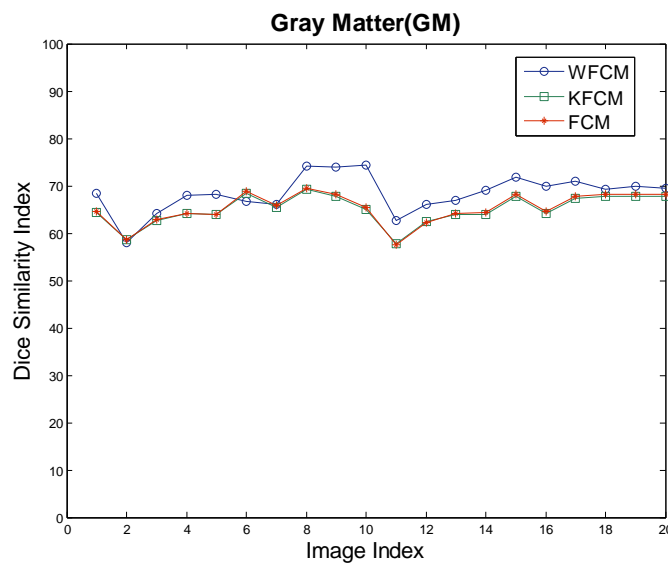

(a) white matter; (b) gray matter.

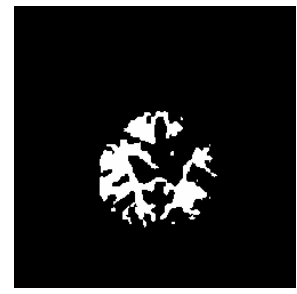

(c)

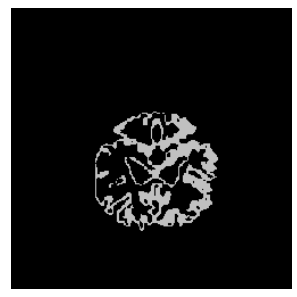

(d)

Figure 9: Segmentation results of the simulated images with proposed algorithm: (a) original image (b) segmented images (c) White Matter (d) Gray Matter.

\section{CONCLUSION}

This paper presents a robust and efficient approach for (the) image segmentation of noisy medical images. The proposed approach makes use of wavelet (DUB4) with Fuzzy C-Means clustering with for the segmentation of noisy medical images. The presented approach has been found robust against various noise levels The experimentation with synthetic BrainWeb images and real images IBSR images have demonstrated the efficiency and robustness of the proposed approach in segmenting noisy medical (MRI) as well as real images as compared to the existing approach. For future works, There are several ways to improve the overall segmentation performance, as preprocessing step for denoising MR images or instead of using conventional FCM with another version of FCM.

\section{REFERENCES}

[1] K. Xiao, S. Hock, and A. Bargiela, "Automatic brain MRI segmentation scheme based on feature weighting factors selection on fuzzy c-means clustering algorithms with Gaussian smoothing," International Journal of Computational Intelligence in Bioinformatics and Systems Biology, vol. 1, pp. 316-331, 2010.

[2] N. Sharma and L. Aggarwal, "Automated medical image segmentation techniques," Journal Medical Physics, vol. 35, pp. 3-14, 2010.

[3] J. Rogowska, "Overview and Fundamentals of Medical Image Segmentation," Handbook of Medical Imaging: Processing and Analysis Management, I. Bankman, Ed, pp. 69-85, 2009.

[4] S. Angenent, E. Pichon, and A. Tannenbaum, "Mathematical Methods in Medical Image Processing,"
Bulletin of the American Mathematical Society, vol. 43, pp. 365-296, 2006.

[5] S. Fiorentini, I. Larrabide, and J. Marcelo. (20/6/2010). A simple $3 D$ image segmentation techniques over medical data.

Available: http://www.frcu.utn.edu.ar/deptos/depto_3/32JAIIO/sis/s is.html

[6] K. Chuang, H. Tzeng, S. Chen, J. Wu, and J. Chen, " Fuzzy c-means clustering with spatial information for image segmentation.," Computerized Medical Imaging and Graphics vol. 30, pp. 9-15, 2006.

[7] K. Sikka, N. Sinha, P. Singh, and A. Mishra, "A fully automated algorithm under modified FCM framework for improved brain MR image segmentation," Magnetic Resonance Imaging, vol. 27, pp. 994-1004, 2009.

[8] J. Jan, Medical Image Processing (Signal Processing and Communications): CRC Press, 2006.

[9] D. Feng, "Segmentation of Soft Tissues in Medical Images," P.h.D thesis National University of Singapore, 2005.

[10] O. wirjadi, "Survey of 3D image segmentation methods," Technical report, 2007.

[11] L. Morra, S. Delsanto, and F. Lamberti, Methods for Neural-Network-Based Segmentation of Magnetic Resonance Images: John Wiley \& Sons, Inc., 2006.

[12] D. Withey and Z. Koles, "Medical image segmentation: Methods and software," Noninvasive Functional Source Imaging of the Brain and Heart and the International Conference on Functional Biomedical Imaging, 6th International Symposium, pp. 140-143, 2007.

[13] K. Wong, "Medical Image Segmentation: Methods and Applications in Functional Imaging," Handbook of Biomedical Image Analysis, , vol. II ; Segmentation Models Part B,, pp. 111-182, 2005.

[14] J. Sijbers, P. Scheunders, M. Verhoye, V. Linden, D. V. Dyck, and E. Raman, "Watershed-based segmentation of 
3D MR data for volume quantization," Journal of Magnetic Resonance Imaging, vol. 15, pp. 679-688, 1997.

[15] L. Zhong, "Stochastic Segmentation Method For Interesting Region Detection And Image Retrieval," P.h.D thesis, North Carolina University at Charlotte 2009.

[16] M. Kass, M.Witkin, and D. Terzopoulos, "Snakes: active contour models," International Journal of Vision, vol. 1, pp. 321-331, 1987.

[17] P. F. Chen, "Image Segmentation/Registration: a Variational Framework for 2-D and 3-D Applications," Ph.D thesis , North Carolina State University, 2009.

[18] A. F. Goldszal and D. L. Pham, "Volumetric Segmentation of Magnetic Resonance Images of the Brain," in Handbook of Medical Image processing, I. Bankman, Ed., ed: Academic Press, 2000, pp. 185-194.

[19] J. Solomon, "Computer-assisted segmentation and tracking of brain lesions in magnetic resonance image based on probabilistic in space and time," M.sc thesis binghamton university, 1993.

[20] S. Luo, "Automated Medical Image Segmentation Using a New Deformable Surface Model," IJCSNS International Journal of Computer Science and Network Security, vol. 6, pp. 109-115, 2006.

[21] D. Pham, C. Xu, and J. Prince, "Current methods in medical image segmentation," Annual Review of Biomedical Engineering, pp. 315-337, 2000.

[22] D. Pham, C. Xu, and L. Prince, "A Survey of Current Methods in Medical Image Segmentation," Annual Review of Biomedical Engineering, vol. 2, pp. 315-338, 1998.

[23] J. Wang, J. Kong, L. Yinghua, Q. Miao, and B. Zhang, "A modified FCM algorithm for MRI brain image segmentation using both local and non-local spatial constraints," Computerized Medical Imaging and Graphics, vol. 32, pp. 685-698, 2008.

[24] C. Dunn, "A Fuzzy Relative of the ISODATA Process and Its Use in Detecting Compact Well-Separated Clusters," Journal of Cybernetics, vol. 3, pp. 32-57, 1973.

[25] J. C. Bezdek, "Pattern Recognition with Fuzzy Objective Function Algoritms," Plenum Press, New York, 1981.

[26] J. Yu and Y. Wang, "Molecular Image Segmentation Based on Improved Fuzzy Clustering," Journal of Biomedical Imaging, vol. 2007, p. 9 pages, 2008.

[27] D. L. Pham, "spatial models for fuzzy clustering. ," Computer Vision and Image Understanding vol. 84, pp. 285-97, 2001.

[28] S. Y. M Ahmed, N Mohamed, A Farag,T Moriarty "A modified fuzzy c-means algorithm for bias field estimation and segmentation of MRI data.," IEEE
Transaction on Medical Imaging, vol. 21, pp. 193-200., 2002.

[29] A. Liew and H. Yan, "An adaptive spatial fuzzy clustering algorithm for 3D MR image segmentation," IEEE Transaction on Medical Imaging, vol. 22, 2003

[30] W. Cai, S. Chen, and D. Zhang, "Fast and robust fuzzy cmeans clustering algorithms incorporating local information for image segmentation.," Pattern Recognition, vol. 40, pp. 825-838, 2007.

[31] J. Wang, J. Kong, L. Yinghua, Q. Miao, and B. Zhang, "A modified FCM algorithm for MRI brain image segmentation using both local and non-local spatial constraints," Computerized Medical Imaging and Graphics, vol. 32, pp. 685-698, 2008.

[32] K.Sikka, N. Sinha, K. Singh, and K. Mishra, "A fully automated algorithm under modified FCM framework for improved brain MR image segmentation " Magnetic Resonance Imaging, vol. 27, pp. 994-1004, 2009.

[33] D.-Q. Zhang and S.-C. Chen, "Clustering Incomplete Data Using Kernel-Based Fuzzy C-means Algorithm," Neural Process. Lett., vol. 18, pp. 155-162, 2003.

[34] D. Zhang and C. Chen, "Robust image segmentation using FCM with spatial constraints based on new kernelinduced distance measure," IEEE Trans. Systems Man Cybernet, vol. 34 pp. 1907-1916, 2004.

[35] D. Zhang and C. Chen, "A novel kernelized fuzzy cmeans algorithm with application in medical image segmentation. ," Artificial Intelligence in Medicine, vol. 32, pp. 37-50, 2004.

[36] S. Kannana, A. Sathyab, S. Ramathilagam, and R. Devi, "Novel segmentation algorithm in segmenting medical images," The Journal of Systems and Software, vol. 83, pp. 2487-2495, 2010.

[37] E.Zanaty, S. Aljahdali, and N. Debnath, "Improving Fuzzy Algorithms For Automatic Magnetic Resonance Image Segmentation," Proceedings of seventeenth International Conference of Software Engineering and Data Engineering, Los Angeles, California, USA, pp. 6066, 2008.

[38] E.Zanaty, S. Aljahdali, and N. Debnath, "A kernelized fuzzy c-means algorithm for automatic magnetic resonance image segmentation," J. Comp. Methods in Sci. and Eng., vol. 9, pp. 123-136, 2009.

[39] E.Zanaty, S. Aljahdali, and N. Debnath, "Improving Fuzzy Algorithms for Automatic Magnetic Resonance Image Segmentation," The International Arab Journal of Information Technology, vol. 7, pp. 271-279, 2010.

[40] M. Yang and H. Tsai, "A Gaussian kernel-based fuzzy cmeans algorithm with a spatial bias correction," Pattern Recognition Letters, vol. 29 pp. 1713-1725, 2008.

[41] BrainWeb: Simulated Brain Database (SBD) http://brainweb.bic.mni.mcgill.ca/brainweb/. 